\title{
Asma bronquial y síndrome de apnea/hipopnea obstructiva del sueño: ¿el nuevo "síndrome de superposición alternativo"?
}

\section{Bronchial asthma and obstructive sleep apnea-hypopnea syndrome: the new "alternative overlap syndrome"?}

Patricia Hidalgo M., MD. ${ }^{(1)}$; Andrea P. Cortés, H., MD. ${ }^{(2)}$; LaURa J. Ruíz S. ${ }^{(3)}$

\section{Resumen}

El sueño es un tema apasionante, actualmente se están entendiendo muchos procesos que tienen lugar desde el punto de vista fisiológico durante el sueño. Se sabe que las crisis de asma tienden a empeorar durante la noche y que generan incremento del riesgo de mortalidad. En estudios epidemiológicos de corte trasversal la prevalencia de hipersomnolencia, ronquido y apnea ha sido mayor en los pacientes con asma. En pacientes con asma de difícil control se ha encontrado que el 74,5\% tienen SAHOS y se sugiere que todos los pacientes con asma de difícil tratamiento eventualmente deben ser evaluados para SAHOS. Parece ser que la prevalencia del síndrome de sobreposición, asma y apnea/hipopnea del sueño es especialmente alta en pacientes con formas más severas de asma y actualmente independiente de los factores de confusión, el asma bronquial ha emergido como un factor de riesgo importante para desarrollar ronquido habitual. Se describen los factores de riesgo y fisiopatología para el síndrome de sobreposición, los efectos del asma sobre el SAHOS, los efectos del SAHOS sobre el asma, los desenlaces clínicos y las propuestas de tratamiento de este síndrome de sobreposición. Se insiste que se requiere un alto índice de sospecha para detectar la sobreposición, se recomienda la evaluación y el tratamiento individualizado encaminado a controlar factores desencadenantes de ambas enfermedades.

Palabras clave: asma, apnea/hipopnea del sueño, síndrome de sobreposición.

\begin{abstract}
Sleep is a fascinating subject. Many processes that take place during sleep are currently been understood from the physiological standpoint. It is known that asthma crises tend to worsen during the night, and that this increases the risk of mortality. In cross-cut epidemiological studies, the prevalence of hypersomnia, snoring, and apnea has been greater in patients with asthma. In patients with difficult to control asthma, it has been found that $74,5 \%$ have SAHOS; it has been suggested that all patients with difficult to control asthma should be evaluated for SAHOS. It seems that the prevalence of asthma and sleep apnea/hypopnea overlap syndrome is especially high in patients with severer forms of asthma. Currently, leaving aside confusing factors, bronchial asthma has emerged as an important risk factor to develop habitual snoring. We describe the risk factors and the pathophysiology of overlap syndrome, the effects of asthma on SAHOS, the effects of SAHOS on asthma, the clinical outcomes, and the suggested treatment of this overlap syndrome. We insist on the fact that a high index of clinical suspicion is required for detecting the overlap. We recommend individualized assessment and treatment in order to control the triggering factors of both diseases.
\end{abstract}

Keywords: asthma, sleep apnea/hypopnea syndrome, overlap syndrome.

\footnotetext{
(1)Internista Neumóloga-Somnóloga, MSc. Hospital Universitario San Ignacio, Pontificia Universidad Javeriana. Bogotá, Colombia.

${ }^{(2)}$ Médico General, Pontificia Universidad Javeriana. Bogotá, Colombia.

${ }^{(3)}$ Estudiante Semillero AOS Pontificia Universidad Javeriana. Bogotá, Colombia Correspondencia: Patricia Hidalgo M., correo electrónico: phidalgo@husi.org.co Recibido: 06/05/15. Aceptado: 12/05/15.
} 


\section{Introducción}

El sueño es un tema apasionante y en la actualidad se están entendiendo muchos procesos que tienen lugar desde el punto de vista fisiológico cuando se duerme, más aún, es pertinente resaltar su relevancia teniendo en cuenta que prácticamente una tercera parte de la vida la dedicamos a dormir.

Se sabe que el sueño tiene un impacto sobre la ventilación y el intercambio gaseoso mediado por el incremento de la resistencia de la vía aérea y la disminución en el volumen pulmonar y en la distensibilidad toracopulmonar (1).

Durante el sueño, el control de los músculos de la vía aérea superior (VAS) y la estabilidad de la vía aérea se encuentran alterados, hecho que al comprometer la anatomía de la VAS, puede desencadenar trastornos obstructivos de la respiración (1).

Se sabe que las crisis de asma tienden a empeorar durante la noche, y que generan incremento del riesgo de mortalidad, con $70 \%$ de muertes y $80 \%$ de paro respiratorio causado por asma durante las horas nocturnas. De igual forma, se ha reconocido un patrón circadiano en la función pulmonar, específicamente en el $\mathrm{VEF}_{1}$ y la relación $\mathrm{VEF}_{1} / \mathrm{CVF}$ independiente de la etapa del sueño; sin embargo, se está a la espera de más investigaciones al respecto (1).

En resumen, se han propuesto factores fisiopatológicos que podrían explicar el asma nocturna: la resistencia aumentada de la vía aérea, los flujos disminuidos, la disminución de la actividad muscular inspiratoria y de la capacidad residual funcional, el enfriamiento de la vía aérea, el incremento de la hiperreactividad bronquial y del tono parasimpático, la inflamación bronquial y el incremento de la histamina circulante (2).

Habitualmente los pacientes con asma se quejan de despertares frecuentes con pobre calidad de sueño, baja eficiencia de sueño y menor porcentaje de sueño profundo. Adicionalmente, se ha encontrado que cuando hay variación circadiana del pico flujo espiratorio mayor al $20 \%$, hay peor desempeño cognitivo diurno (1).

\section{Síndrome de sobreposición}

En la literatura médica se ha acuñado el término OLDOSA, su sigla en inglés por "obstructive lung disease and obstructive sleep apnea", y se refiere a la coexistencia bien sea de enfermedad pulmonar obstructiva crónica o asma bronquial con el síndrome de apnea obstructiva del sueño.

De igual manera, se ha propuesto un término específico para la coexistencia de asma bronquial y síndrome de apnea obstructiva del sueño, "alternative overlap syndrome".

La coexistencia de estas dos enfermedades hace que cada trastorno sea influenciado de forma deletérea por el otro.

Existe también asociación del asma con otras enfermedades como reflujo gastroesofágico, rinosinusitis, obesidad y trastornos mentales.

\section{Epidemiología}

\section{Riesgo de SAHOS en pacientes asmáticos}

En estudios epidemiológicos de corte transversal, la prevalencia de hipersomnolencia, ronquido y apnea ha sido mayor en los pacientes con asma (3).

En el Community Health Respiratory Survey en el Reino Unido, se encontró que personas con asma de todas las edades y pesos roncaban con mayor frecuencia que los controles (29\% vs. 9\%). Así mismo, en los europeos, el autorreporte de ronquido habitual y apnea fue significativamente más frecuente en personas con asma comparados con los controles sin asma ( $14 \%$ vs. $9 \%$ y $3 \%$ vs. $1 \%$, respectivamente), independientemente de su edad, peso corporal, sexo o tabaquismo (4).

Se han realizado estudios buscando la asociación prospectiva del asma bronquial con el desarrollo incidente de SAHOS evaluado con polisomnograma. En el estudio del Wisconsin Sleep Cohort Study se encontró que el asma bronquial preexistente es un factor de riesgo para el desarrollo de SAHOS clínicamente relevante y la asociación fue dependiente de la duración del asma bronquial. El riesgo relativo ajustado fue de 1,39 (IC95\% 1,06-1,82), controlando 
por sexo, edad, el IMC y otros factores. También se encontró asociación entre el asma y el SAHOS de reciente inicio con hipersomnolencia habitual (RR: 2,72; IC95\% 1,26-5,89; p=0,04). La duración del asma fue relacionada con ambos, SAHOS incidente (riesgo relativo: 1,07 por cada 5 años de incremento en la duración del asma (IC95\% 1,02-1,13; $\mathrm{p}=0,01$ ) y SAHOS incidente con hipersomnia habitual (RR: 1,18; IC95\% 1,07-1,31; $\mathrm{p}=0.02)$ (3).

En la misma cohorte, el riesgo predicho de desarrollar SAHOS en pacientes con asma de inicio en el adultez y en la infancia fue OR de 1,48 y 2,34, respectivamente; es importante anotar que cuando se tiene en cuenta la duración del asma y se crea una unidad de tiempo "5 años de duración del asma", se correlaciona con $10 \%$ de incremento en el riesgo (4).

En otra cohorte de asma, la incidencia global de SAHOS fue 2,51 veces mayor (12,1 versus 4,84 por 1.000 personas-año) comparada con los no asmáticos. El hazard ratio (HR) ajustado de SAHOS se incrementó a 1,78 para los pacientes asmáticos con una o menos visitas a urgencias y a 23,8 para aquellos que acudieron al servicio de urgencias más de una vez en el año. Adicionalmente, el HR ajustado en pacientes con tratamiento esteroide inhalado comparado con los pacientes sin tratamiento fue 1,33 (IC95\% 1,01-1,76). En conclusión, los pacientes con asma tienen un riesgo significativamente mayor de desarrollar SAHOS que la población general. Los resultados sugieren que el riesgo es proporcional al control del asma y que los pacientes que reciben tratamiento con esteroides inhalados tienen un riesgo mayor de desarrollar SAHOS que los que no lo reciben. Estos HR fueron mayores en hombres que en mujeres $(2,14$ vs. 1,54) y en aquellos con alguna comorbilidad comparados con los pacientes sin comorbilidades $(2,05$ vs. 1,90) (5).

En una institución estadounidense se encuestaron pacientes en un servicio de asma y de Medicina interna para analizar el riesgo de SAHOS, mediante el cuestionario de Berlín (valor predictivo positivo de 0,89). Se determinó que el riesgo era mayor en los asmáticos $(39,5 \%)$ comparado con los del grupo de Medicina interna $(27,2 \% ; p=0,04)(1)$.
En una cohorte canadiense de pacientes con asma con criterios de severidad de la ATS, y definiendo SAHOS por índice de apnea e hipopnea por hora (IAH) de 15 o más, se encontró que el $88 \%$ de los pacientes con asma severa, el $58 \%$ de los que tenían asma moderada y el $31 \%$ de los pacientes sin asma, cumplían criterios para SAHOS (1).

Por otra parte, un estudio en Portugal ha reportado prevalencia del $57,4 \%$ de SAHOS en pacientes asmáticos sometidos a estudio de sueño, valor que es de hecho mayor que en la población general (6).

Braido et al. han investigado el riesgo de SAHOS en los pacientes con asma y en aquellos con asma acompañada de rinitis alérgica, encontrando valores de $47,3 \%$ y $55,9 \%$ respectivamente, con un riesgo global de 52,6\%. La rinitis se asoció con un OR 1,44 veces más alto de riesgo de SAHOS. La duración de la rinitis y su severidad también fueron factores para el incremento en el riesgo de SAHOS. Con base en los resultados expuestos, se propone que la presencia de asma, concomitante con rinitis, se asocia con mayor riesgo de SAHOS, independiente de la presencia de obesidad y de otros factores (7).

En los pacientes con asma de difícil control, se ha encontrado que el 74,5\% tienen SAHOS. Con base en todo lo anterior, se sugiere que todos los pacientes con asma de difícil tratamiento sean evaluados para SAHOS (8). Es más, se ha demostrado que el riesgo de SAHOS se asocia con el empeoramiento de los índices de control de asma, el incremento de la utilización de los sistemas de salud y el deterioro de la calidad de vida. A pesar de que presentan una duración similar de sueño, los pacientes con asma severa y no severa tienen calidad peor de sueño y más somnolencia diurna que la población general. Es así que los síntomas de SAHOS son más prevalentes entre los asmáticos y se sugiere que hay una relación bidireccional entre SAHOS y asma (9).

Finalmente, después de ajustar por covariables, el SAHOS diagnosticado permanece como un predictor de síntomas diurnos persistentes (OR 2,08) pero no de síntomas nocturnos y, de manera similar, en el análisis univariado, el uso de presión continua positiva en la vía aérea (CPAP) estuvo asociado 
con riesgos menores de síntomas diurnos (OR 0,5; $\mathrm{p}=0,049$ ) pero no de síntomas nocturnos (4).

\section{Asma en síndrome de apnea/hipopnea del sueño}

La prevalencia de asma bronquial en pacientes con y $\sin$ SAHOS, es de $10,4 \%$ y $5,1 \%(\mathrm{p}<0,0001)$, respectivamente, mientras que la de asma asociada a EPOC es de 3,3\% y 0,9\% (p<0,0001) (10).

Por lo anterior, parece ser que la prevalencia del síndrome de sobreposición, es especialmente alta en los pacientes con formas más severas de asma.

\section{Fisiopatología y factores de riesgo para el síndrome de sobreposición}

Independientemente de los factores de confusión, el asma bronquial ha emergido como un factor de riesgo importante para desarrollar "ronquido habitual" (3).

Existen numerosos factores fisiopatológicos $\mathrm{y}$ clínicos que contribuyen a esta sobreposición, y las interacciones complejas entre estas dos condiciones deben tener en cuenta mecanismos etiopatogénicos específicos y endotipos de enfermedad diferentes. La existencia del endotipo asma-SAHOS está apoyada en varios argumentos de interacción dual, vías biológicas comunes y respuestas terapéuticas únicas (4).

Las interacciones bidireccionales entre el asma bronquial y el SAHOS pueden ser clasificadas como efectos directos e indirectos, mecánicos, neurales o biológicos por naturaleza.

\section{Efectos del asma sobre el SAHOS}

\section{Efectos directos}

Existen varias teorías (4) al respecto:

- Teoría de la arquitectura del sueño: cambios en la reactancia durante el sueño REM (movimientos oculares rápidos), confirman que los pulmones se hacen más rígidos con el sueño, particularmente en los pacientes con asma (4).

- Teoría del tirón traqueal: las personas con asma tienen mayores reducciones en los volúmenes pulmonares durante el sueño, especialmente en el REM, lo cual atenúa el efecto de endurecimiento del tirón traqueal sobre el segmento de la VAS faríngea, lo cual se asemeja al efecto de la posición yacente o la obesidad abdominal (4).

- Teoría de la interdependencia de la fase respiratoria: el asma se caracteriza por la limitación al flujo espiratorio y el incremento de la resistencia de la vía aérea durante la exhalación. Esto puede predisponer a mayor colapsabilidad de la VAS durante la inspiración en el sueño (4).

- Teoría inflamatoria: el asma se asocia con inflamación aguda y crónica, lo cual puede afectar la fuerza de los músculos respiratorios, incluyendo los dilatadores de la VAS (4).

\section{Efectos indirectos}

- Efectos de los corticosteroides: el depósito de grasa en y alrededor de la VAS, es resultado de su absorción sistémica así como de miopatía de los músculos dilatadores de la VAS, acumulación de líquido en el cuello y obesidad (4).

Los corticoides inhalados son los medicamentos más efectivos y más utilizados en el asma. Sin embargo, sus efectos a largo plazo sobre la colapsabilidad de la faringe se desconocen. En clínicas de asma, donde los pacientes requieren utilizar frecuentemente corticosteroides orales, se ha encontrado alta prevalencia de SAHOS (mayor a 90\%) después de ajustar por el IMC y la circunferencia de cuello (1).

- Enfermedad nasal: el área transversal y el volumen de la vía aérea nasal es más pequeña en las personas con asma, independientemente de su condición alérgica (4).

Muchos de los pacientes con asma no atópica y la mayoría de los pacientes con asma y componente atópico presentan obstrucción nasal debido a rinitis y a sinusitis crónica. Lo anterior se debe a una reducción del calibre de la vía aérea generada por la congestión nasal, la resistencia al flujo de aire y los pólipos nasales, factores que llevan a incremento en la presión negativa intratorácica y 
faríngea, promoviendo de esta manera el colapso de la vía aérea durante la inspiración, el ronquido y la apnea obstructiva.

Así mismo, en pacientes con asma crónica, la inflamación persistente de la mucosa afecta la vía aérea superior y disminuye el área transversa de la faringe, promoviendo el colapso de la vía aérea (1).

- Consumo de cigarrillo: es posible que aparezca el endotipo de asmático fumador.

\section{Efectos del SAHOS sobre el asma}

\section{Efectos directos}

- Teoría mecánica: el SAHOS incrementa la carga resistiva sobre la vía aérea inferior, hecho impuesto a una vía aérea de por sí ya desafiada especialmente durante el sueño (4).

La presión negativa intratorácica durante los eventos obstructivos lleva a pérdida intermitente del tono del esfínter esofágico inferior, en tanto que el reflujo gastroesofágico asociado con microaspiración bronquial de ácido gástrico, promueve potencialmente el asma nocturna (1).

- Teoría del reflejo neural: el cierre de la VAS y el ronquido pueden desencadenar broncoconstricción mediada vagalmente y empeorar la hiperreactividad de la vía aérea (4).

El incremento del tono vagal durante la apnea obstructiva puede contribuir al asma nocturna a través de receptores muscarínicos de las vías aéreas centrales y superiores (1).

- Teoría de la arquitectura del sueño: la privación y fragmentación del sueño causadas por el SAHOS también puede exacerbar el asma nocturna al incrementar la resistencia de la vía aérea y anular el despertar en respuesta a la broncoconstricción (4).

- Teoría de la hipoxia intermitente: en pacientes con SAHOS, las oclusiones repetitivas de la vía aérea durante el sueño llevan a hipoxia intermitente y reoxigenación, además de la producción subsecuente de especies reactivas de oxígeno y desencadenamiento de la cascada de estrés oxidativa, inflamación, recargas del tono simpático y disfunción endotelial. Todo lo anterior, puede contribuir al fenotipo inflamatorio no eosinofílico de pacientes con asma de difícil control (4).

También, por estimulación de los receptores en los cuerpos carotídeos, la hipoxia intermitente puede incrementar la respuesta bronquial a través de vías vagales (1).

- Teoría de la inflamación local: el SAHOS se asocia con la inflamación de la VAS, lo cual puede tener una influencia potencial sobre las vías aéreas inferiores (4).

- Teoría de inflamación sistémica: adicionalmente, la privación del sueño y la obesidad generan diátesis inflamatoria. El SAHOS podría causar estrés oxidativo e inflamación de la vía aérea inferior y así los efectos proinflamatorios de un trastorno podrían influir en la expresión del otro. El factor de necrosis tumoral (FNT) se encuentra elevado en SAHOS, independientemente del peso corporal, y en algunos casos puede desempeñar un rol en la patogénesis del asma (4).

- Teoría vascular: el factor de crecimiento endotelial vascular (VEGF) puede tener un papel importante en la patogénesis del asma. El SAHOS tiene concentraciones elevadas de VEGF que se correlacionan con la severidad de la enfermedad, determinada por el IAH y el grado de desaturación nocturna (4).

- Teoría de la leptina: los pacientes con SAHOS tienen niveles séricos elevados de leptina, cuyos efectos proinflamatorios sugieren que esta hormona puede ser relevante en las exacerbaciones de asma (4).

- Teoría de la disfunción cardíaca: el SAHOS induce disfunción cardiaca, que a su vez podría causar obstrucción de la vía aérea (4).

\section{Efectos indirectos}

- Enfermedad nasal

- Consumo de cigarillo: se considera al tabaquismo como un factor de riesgo independiente para 
SAHOS y para reflujo gastroesofágico. Consumir tabaco o marihuana puede desencadenar síntomas como sibilancias, tos y producción de esputo (4).

A pesar de que los fenotipos asma-obesidad y endotipos han sido descritos recientemente, sería interesante estudiar de manera sistemática la contribución de los trastornos respiratorios del sueño a todos ellos (4).

Contrario a una teoría previa, estudios recientes han encontrado que el esfínter esofágico inferior se contrae durante los episodios de apnea, inhibiendo así el reflujo del contenido gastroduodenal hacia el esófago. Las relajaciones transitorias del esfínter (posiblemente asociadas con un alertamiento inducido por la deglución y el peristaltismo esofágico) parecen estar más relacionadas con el mecanismo de reflujo en los pacientes con SAHOS (4).

\section{Evidencia}

Los pacientes con asma severa que tienen SAHOS leve concomitante, tienen aumento de la proporción de neutrófilos en el esputo, menor número de macrófagos y cambios en la remodelación de la vía aérea, comparados con los asmáticos sin SAHOS. Así mismo, niveles elevados de interleucina (IL-8) y metaloproteinasa 9 (MMP-9) en el esputo y la membrana basal bronquial más delgada se han correlacionado de manera inversa con el IAH (11).

También se ha encontrado mayor prevalencia de hipertensión arterial y enfermedades cerebrovasculares en pacientes asmáticos severos con SAHOS, hallazgo que podría estar relacionado con la inflamación neutrofílica asociada al SAHOS (11).

Lo anterior puede señalar un fenotipo específico de asma neutrofílica que debe ser identificado para orientar el tratamiento. En efecto, incrementar las dosis de los esteroides puede tener un efecto deletéreo en el contexto de la inflamación neutrofílica/no eosinofílica, que debe tenerse en cuenta en este contexto. Los esteroides contribuyen a la infiltración neutrofílica de la vía aérea a través de la disminución de la apoptosis de los neutrófilos. Lo anterior podría llevar a un aumento en el riesgo de SAHOS en esta población y la severidad de la enfermedad. El SAHOS contribuye a la inflamación neutrofílica y por tanto a la resistencia a los esteroides y la severidad del asma, llevando a un círculo vicioso (11).

Por su parte, la obesidad es un factor de riesgo para asma y SAHOS. Existe un efecto proporcional al incrementar el IMC sobre el riesgo del asma incidente, especialmente en mujeres (1).

En conclusión, los mecanismos potenciales por los cuales el SAHOS puede empeorar el asma son multifactoriales, sabiendo que los episodios apneicos obstructivos están asociados con alertamientos repetitivos durante el sueño, alteración de la actividad del sistema autonómico e hipoxemia intermitente (1).

\section{Desenlaces clínicos y tratamiento del síndrome de sobreposición}

No hay estudios a largo plazo que hayan evaluado desenlaces, tales como los despertares nocturnos en estos pacientes.

En la actualidad no existen guías específicas para el manejo del síndrome de sobreposición (1). Parece que el CPAP puede representar un beneficio clínico potencial en el tratamiento del síndrome de sobreposición asma-SAHOS y existen datos que documentan que el tratamiento con CPAP para el SAHOS comórbido mejora los síntomas del asma, disminuye el uso de medicamentos de rescate y optimiza la calidad de vida de los pacientes (1).

En un ensayo clínico aleatorizado a corto plazo, el uso del CPAP disminuyó la reactividad de la vía área en los pacientes asmáticos sin SAHOS, posiblemente debido a la reducción de la inflamación bronquial (1). Sin embargo, en otro estudio se plantea que es probable que la terapia con CPAP incremente la hiperreactividad bronquial en pacientes no asmáticos con SAHOS y lleve a deterioro de su arquitectura del sueño. En vista de los resultados contradictorios en los diferentes estudios clínicos, se requieren estudios prospectivos con mayor tamaño de muestra sobre fenotipos bien definidos, que permitan determinar el rol y las limitaciones de la terapia con CPAP en estos pacientes (12). 
Teniendo en cuenta que la evidencia actual se basa en poblaciones de tamaño pequeño y de características heterogéneas, es difícil emitir conclusiones definitivas acerca del manejo óptimo en pacientes con el síndrome de sobreposición. No obstante, es crucial realizar una evaluación cuidadosa de las comorbilidades presentes y optimizar el tratamiento del SAHOS y el asma (12).

Así mismo, se requieren estudios a largo plazo para determinar la aplicación óptima de CPAP en el paciente con síndrome de sobreposición, que permitan evaluar la mejoría de los síntomas del asma, la necesidad de medicación y los desenlaces cardiorrespiratorios y de calidad de vida (1).

Recientemente, la iniciativa AIRWAYS ICP (del inglés Integrated Care Pathways for Airway Diseases) lanzó una propuesta para desarrollar, de forma multisectorial, estrategias para reducir la carga de enfermedades crónicas respiratorias, incluyendo guías y políticas que utilizan las redes de trabajo y los puntos de vista de todos los actores del sistema de salud. En los pacientes con asma se recomienda que el SAHOS debería investigarse cuando existe control inadecuado de los síntomas nocturnos del asma a pesar de recibir el tratamiento recomendado por las guías. Existe evidencia en la literatura de que el uso de CPAP contribuye al control de la enfermedad en pacientes asmáticos con SAHOS y con asma no controlada (13).

\section{Limitaciones}

Los humidificadores del circuito del CPAP se necesitan para evitar que el aire seco y frío induzca asma y se formen costras. Deben limpiarse para prevenir que los microorganismos se propaguen en el medio tibio y húmedo y desciendan a la vía aérea. Además, Martin y Pak mostraron que los pacientes asmáticos sin apnea, presentaron adherencia baja a la CPAP; de hecho, interrumpió el sueño por ser incómodo para ellos (14).

Los tratamientos de segunda línea para el SAHOS, como los dispositivos de avance mandibular y la cirugía de vía aérea superior, no han sido evaluados de manera prospectiva en este tipo de pacientes. Sin embargo, la cirugía bariátrica en pacientes con SAHOS y obesidad mórbida puede ser efectiva no solamente para mejorar el SAHOS sino el asma.

No hay estudios que evalúen desenlaces clínicos relacionados con el uso de medicamentos para el síndrome de sobreposición. Por el momento, parece que el manejo del asma en pacientes con SAHOS debe realizarse de acuerdo con las guías actuales, adicional a la optimización del tratamiento del SAHOS comórbido (1).

\section{Conclusiones}

- Una vez se presenta SAHOS en pacientes con asma, éste puede impactar de manera negativa los desenlaces relacionados con el asma, afectando así su control diurno y nocturno (3).

- El tratamiento del SAHOS lleva a mejorar los síntomas del asma, el pico espiratorio flujo matutino y la calidad de vida (3).

- Es necesario incluir el SAHOS en las guías actuales de asma (3).

- Se debe evaluar y tratar el SAHOS independiente de la obesidad en los programas de asma (9).

El GINA recomienda investigar su coexistencia en todos los pacientes con asma, especialmente en aquellos con asma severa, de difícil control y asociada con obesidad; en los pacientes con asma nocturna se evaluará la coexistencia (15).

Desde el punto de vista clínico, los pacientes con asma deben ser evaluados de manera proactiva buscando la coexistencia de un trastorno respiratorio del sueño y de igual manera, los pacientes con SAHOS deben ser evaluados para asma, dado que hay dificultad para diferenciar algunos de los síntomas nocturnos (4).

Hay factores potencialmente efectivos en la disminución del riesgo y la severidad del SAHOS en pacientes con asma, tales como la pérdida de peso, la posición decúbito lateral al dormir y la suspensión del tabaquismo (1). 
El efecto del ajuste de los medicamentos utilizados para el asma bronquial sobre la mejoría del SAHOS no ha sido estudiado (1).

En pacientes no asmáticos con SAHOS y con rinitis concomitante, hay evidencia de que los corticosteroides inhalados disminuyen la inflamación de la vía aérea superior y mejoran el IAH (1).

Para finalizar y mencionar datos de nuestra población, en una muestra de 6.200 pacientes llevados a polisomnograma en el Hospital Universitario San Ignacio, se encontró que el 5\% tenía asma bronquial y de estos el 75\% tenía SAHOS en forma concomitante. Estos resultados preliminares confirman la necesidad de tener alta sospecha de la coexistencia de ambas enfermedades y estar atentos a orientar su manejo de la mejor forma.

\section{Resumen}

1- El SAHOS y el asma son trastornos respiratorios prevalentes que a menudo se sobreponen.

2- Se requiere un alto índice de sospecha para detectar la sobreposición.

3- Se recomienda tratamiento individualizado y encaminado a controlar factores desencadenantes de ambas enfermedades.

4- Factores agravantes: ganancia de peso, rinitis, sinusitis, pólipos nasales, inflamación de la vía aérea superior, uso de corticosteroides.

5- Factores protectores: pérdida de peso, postura corporal al dormir, uso de broncodilatadores y uso de corticosteroides inhalados.

\section{Futuro}

1- Identificación de fenotipos y factores genéticos.

2- Determinación del papel y limitaciones de las terapias estándar para SAHOS y asma.

3- Identificación de biomarcadores para uso clínico.

\section{Conflictos de interés}

Los autores declaran no tener conflictos de interés.

\section{Bibliografía}

1. Kryger, MH, Roth, T, Dement, WC. Principles and practice of sleep medicine. Sixth edition. Principles and Practice of Sleep Medicine. Elsevier Health Sciences; 2016.

2. Khan WH, Mohsenin V, D'Ambrosio CM. Sleep in asthma. Clinics in chest medicine. 2014;35 (3):483-93.

3. Teodorescu M, Barnet JH, Hagen EW, Palta M, Young TB, Peppard PE. Association between asthma and risk of developing obstructive sleep apnea. JAMA. 2015;313 (2):15664.

4. Puthalapattu S, Ioachimescu OC. Asthma and Obstructive Sleep Apnea. J Invest Med. 2014;62(4):665-75.

5. Shen TC, Lin CL, Wei CC, Chen CH, Tu CY, Hsia TC, et al. Risk of obstructive sleep apnea in adult patients with asthma: a population-based cohort study in Taiwan. PloS one. 2015;10(6):e0128461.

6. Madama D, Silva A, Matos MJ. Overlap syndrome-Asthma and obstructive sleep apnea. Rev Portuguesa Pneumol. English Edition. 2015.

7. Braido F, Baiardini I, Lacedonia D, Facchini FM, Fanfulla F, Molinengo G, et al. Sleep apnea risk in subjects with asthma with or without comorbid rhinitis. Respiratory care. 2014;59 (12):1851-6.

8. Guven SF, Dursun AB, Ciftci B, Erkekol FO, Kurt OK. The prevalence of obstructive sleep apnea in patients with difficult-to-treat asthma. Asian Pacific J Allerg Immunol. 2014;32 (2):153.

9. Teodorescu M, Broytman O, Curran-Everett D, Sorkness RL, Crisafi G, Bleecker ER, et al. Obstructive sleep apnea risk, asthma burden, and lower airway inflammation in adults in the Severe Asthma Research Program (SARP) II. J Allerg Clin Immunol. In Practice. 2015;3(4):566-75.

10. Greenberg-Dotan S, Reuveni H, Tal A, Oksenberg A, Cohen A, Shaya FT, et al. Increased prevalence of obstructive lung disease in patients with obstructive sleep apnea. Sleep and Breathing. 2014;18(1):69-75.

11. Taillé C, Rouvel-Tallec A, Stoica M, Danel C, Dehoux M, Marin-Esteban V, et al. Obstructive sleep apnoea modulates airway inflammation and remodelling in severe asthma. PloS one. 2016;11(3):e0150042.

12. Prasad B, Nyenhuis SM, Weaver TE. Obstructive sleep apnea and asthma: associations and treatment implications. Sleep Med Rev. 2014;18 (2):165-71.

13. Bousquet J, Cruz AA, Robalo-Cordeiro C. Obstructive sleep apnoea syndrome is an under-recognized cause of uncontrolled asthma across the life cycle. Rev Portuguesa Cardiol. 2016;22(1):1-3.

14. Qiao YX, Xiao Y. Asthma and obstructive sleep apnea. Chinese Med J. 2015;128(20):2798.

15. Pocket guide for asthma management and prevention GINA updated 2016; [Acceso Abr 2016]. 